\title{
Contents, Vol. 3, 1950
}

\section{ONCOLOGIA}

Zeitschrift fiir Erforschung, Bekampfung, Behandlung und Soziologie der Krebskrankheit Journal of Cancer Research, Prevention, Treatment and Sociological Aspect

Revue de I'Exploration, du Traitenient et de la Sociologie du Cancer

Rivista per la Ricerca, il Trattamento e la Sociologia del Cancro Offizielles Organ der Schweiz. Nationalliga fiir Krebsbekiimpfung. Organe officie! de la Ligue nationale Suisse contre le Cancer. Organo ufficiale della Lega nazionale svizzera contro il Cancro.

\section{EDITORES :}

J. L. Nicod-Lausanne: President der Schweiz. Nationalliga fiir Krebsbekainpfung. P. Jung-St. Gallen: Sekretar der Schweiz. Nationalliga fiir Krebsbekiimpfung. Biologia Generalis:

Paihologia: Medicina Interna: Chirurgia: Gynaecologia: Radiologia: Neurochirurgia: Ophthalmologic.: Otorhinolaryngologic Dermatologia: Paediatria : Sociologia:

A. Grumbach-Ziirich, F. Leuthardt-Ziirich,

A. Vannotti-Lausanne.

J. L. Nicod-Lausanne, E. Uehlinger-St. Gallen.

W. Loffler-Ziirich, M. Roch-Geneve, H. Staub-Basel.

A. Brunner-Ziirich, P. Decker-Lausanne.

E. Held-Ziirich, H. de Watteville-Geneve.

R. Gilbert-Geneve, A. Zuppinger-Bern.

H. Krayenbiihl-Ziirich, A. Jentzer-Geneve.

A. Franceschetti-Geneve, H. Goldmann-Bern.

L. Riiedi-Zurich, E. Oppikofer-Basel.

W. Jadassohn-Geneve, G. Miescher-Ziirich.

E. Freudenberg-Basel, E. Glanzmann-Bern.

A. Koller-Bern, P. Vollenweider-Bern.

Redactor: Hans R. Schinz, Zurich

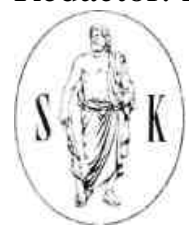

Vol. Ill

1950 


\section{BASEL (Schweiz) S. KARGER NEWYORK}

Alle Rechte vorbehalten

Printed in Switzerland Buchdruckerei Karl Werner AG. in Basel Cliches: Aberegg-Steiner \&

Cie. AG., Ben

INDEX

Attinger. E., Uber die Kombination von Lungenkarzinom mit Lungentuberkulose

Boissonnas, A., vide S. Neulvmm

Caravaylioti, R., vide P. Li Voti

Cocchi, V., und E. Meier. Zur Frage der Therapie des groBfollikularen Lymphoblastoma

Decker, P.. Tactique de 1'exerese pour cancer bronchique

Hoch, A., Rontgenkurabilitat der Sarkome. Ziircher Beobachtungen

an 1113 Sarkomfallen 1919-1948

Hohl, K.. Therapie und Prognose des primaren und sekundaren

osteogenen Sarkoms. Ziircher Erfahrungen 1919-1949

Jeannet, E., Repartition des Substances steroid.es apparentees aux

Hormones sexuelles dans l'Organisme de 1'Animal sain et can-

cereux

Li Voti, P., V. Mutolo, R. Caravaglios, SuH’attivita proteolitica dei sieri normali e neoplastic!

Mannino, R., Sopra un caso di Linfosarcoma della parotide (Con tribute clinico ed anatomo-istologico)

Meier, E.. vide U. Cocchi

Mutolo, V., vide P. Li Voti

Xeukomm, S., et A. Boissonnas, Activite dehydrasique des organes de souris saines et cancereuses

Rigo, J., E. Scipiades und L. Vdczy, Neuere Untersiichungen zur Klarung der Zusammenhange zwischen der glandular-cystischen Hyperplasie und den genitalen Karzinomen

Da Rugna, D., Uber 2 Falle doppelseitiger Nebennieren-Sarkome

mit zentralnervosen Symptomen 219

Sarasin, P., Le plasmocytome. Etudes de 37 cas de Zurich 90

Schild, Ch.A., Doppeltumoren. Beitrag zur Frage der Kombinations-, Induktions- und Kollisionsgeschwiilste 117

Schinz, H. R., Ziircher Erfahrungsn iiber die Krebssterblichkeit seit der Jahrhundertwende 215

Scipiades, E., vide J. Rigo

Steinmann, H., Das Ionogramm bei Krebskranken und bei Krebs-

kachexie 53

Vaczy, L., vide J. Rigo

Werthemenn, A., Uber spongiozytares Adamantinom

Ctesellschaytsbertehte - Society Transactions - \&oci^tes

VI. Italienischer Krebskongrefi 191

liasnistik - Casaisti < ine - Case Report

Cocchi, U., Pancoast-Syndrom. Kasuistische Mitteilung 
Sarasin, Ph., Un cas de Psammo-Adenocarcinome de l’Ovaire. . . . 244 